\title{
Comportamento Adaptativo e Perspectivação do Futuro: Algumas Evidências nos Contextos da Educação e da Saúde
}

\author{
Adaptive Behaviour and Future Time Perspective: Some Evidence \\ from Education and Health Settings
}

\author{
Renato Gil Carvalho*, Margarida Pocinho \& Carla Silva \\ Secretaria Regional de Educação, Funchal, Portugal \& Universidade de Madeira, Funchal, Portugal
}

\begin{abstract}
Resumo
O papel do futuro no comportamento actual dos indivíduos, em particular dos jovens, tem sido alvo de atenção ao longo do tempo, destacando-se aquele que tem vindo a ser um efeito consistente de associação entre, por um lado, a perspectivação e a construção de planos de futuro - expressados pelo conceito de perspectiva temporal de futuro (PTF) - e, por outro, a expressão de comportamentos adaptativos, que contribuem para uma maior adaptação ao meio. Dois dos contextos de estudo em que essa relação é mais abordada são os da educação e da saúde, este último especificamente ao nível da promoção de comportamentos saudáveis e da prevenção de riscos. Tendo em conta estes dois contextos, o presente trabalho pretende sistematizar e discutir um conjunto de dados, resultantes de vários estudos, e que se consideram relevantes para a evidenciação dessa relação.

Palavras-chave: Perspectiva Temporal de Futuro; Motivação; Comportamento Adaptativo.
\end{abstract}

\begin{abstract}
The role of future in the current individual behavior, especially among youngsters, has been receiving considerable attention. It is important to underline the consistent effect of association between future plans construction and perspectives - as expressed by the Future Time Perspective (FTP) concept and adaptive behavior expression. This aspect is considered as a contributor to higher adaptation. Two of the contexts where that relation is studied are education and health, the latter specifically at healthy behavior development and risk prevention levels. Having in mind these two dimensions, the present article intends, to discuss some data from several studies, which are considered rele-vant for giving emphasis on such relation.

Keywords: Future Time Perspective; Motivation; Adaptive Behavior.
\end{abstract}

No âmbito da psicologia, têm vindo a ser desenvolvidos, ao longo do tempo, diversos estudos que enfatizam o papel que o futuro - e o modo como é perspectivado pelos indivíduos - pode ter nos comportamentos que estes apresentam no presente. Apesar desse papel do futuro poder ser operacionalizado de diversos formas, consideramos que a perspectiva temporal de futuro (PTF) é um conceito que se pode designar de aglutinador e tem um grande valor parcimonioso. Não admira, assim, que este conceito tenha sido abordado de diferentes modos e objectivos: desde a sua evolução ao longo do ciclo de vida, até à sua relação com variáveis de cariz sociodemográfico, o estudo da PTF e da construção de planos de futuro tem recolhido vários contributos.

\footnotetext{
*Endereço para correspondência: Secretaria Regional de Educação, Divisão de Apoio Psicológico e de Orientação Escolar e Profissional, Edifício D. João, Rua Cidade do Cabo, n 38, Funchal, Madeira, Portugal, 9050-047. E-mail: renatoggc@gmail.com
}

Ora, passadas várias dezenas de anos desde que Nuttin (1964) publicou um dos primeiros trabalhos com maior notoriedade neste domínio, pretende-se sublinhar ou reiterar o valor adaptativo que a PTF tem no comportamento dos indivíduos, focando, para isso, dois contextos onde se têm vindo a publicar alguns trabalhos: a educação e a saúde. Para tal, apresenta-se o presente texto, que se encontra estruturado em três partes: começa-se por discutir o papel do futuro no comportamento, clarificando-se seguidamente o conceito de PTF nas suas diferentes expressões, para, por fim, proceder-se a uma análise de vários dados produzidos ao longo do tempo, provenientes de diferentes contextos.

\section{O Impacto do Futuro e da Percepção do Tempo no Comportamento}

A percepção do tempo constitui uma das características básicas do comportamento humano e tem sido uma variável psicológica crucial durante a evolução da espé- 
cie humana (Díaz-Morales, 2006). Relativamente ao futuro, caracterizando-se este por ser uma instância de aspirações, de desejos, de medos, de preocupações e de esperanças (Paredes \& Pecora, 2004), assume um papel central no modo como os indivíduos atribuem significado à experiência e agem. O futuro e a representação que dele é feita são, de facto, elementos centrais da vivência psicológica individual de cada um. Na verdade, quase não seríamos capazes de viver sem a memória do passado, uma orientação para o presente e o sentido de devir (Araújo, 2005).

A relação entre o presente e o futuro é uma via de dois sentidos, em que o conhecimento e os estados de espírito actuais podem afectar as construções do futuro por parte dos indivíduos, e as suas construções do futuro podem influenciar os seus estados cognitivos e emocionais presentes (Karniol \& Ross, 1996).

A ideia de que as expectativas das pessoas em relação ao futuro podem ser usadas para entender o seu comportamento, de facto, remonta já a Platão. Foram, no entanto, Frank (1939) e Lewin $(1935,1942)$ dois dos primeiros autores modernos a discutir a importância do futuro imaginado na compreensão da motivação e do comportamento humanos. Olhando para a perspectiva temporal como uma orientação em relação a objectivos futuros, assumiram que a capacidade para ter em conta antecipações de resultados ou consequências de comportamentos, resulta de processos desenvolvimentistas (Frank, 1939; Husman \& Lens, 1999; Lewin, 1935, 1942).

De acordo com Zimbardo e Boyd (1999), a perspectiva temporal é um processo no qual as experiências dos indivíduos são ligadas a moldes temporais ou espaços de tempo. Os resultados anteriores de investigadores como Lewin (1951) e Nuttin (1964), em relação à perspectiva temporal, fortalecem a crença de Zimbardo e Boyd (1999), de que os períodos temporais, nomeadamente passado, presente e futuro, influenciam o modo como o indivíduo organiza e atribui significado às experiências. Estes espaços temporais influenciam também as decisões que os indivíduos tomam em resposta a determinados estímulos (Abousselam, 2005).

Os indivíduos podem fazer planos relevantes para as suas tarefas de vida e são frequentemente guiados por representações distintas de si próprios no futuro (Karniol \& Ross, 1996). Mas, ao passo que alguns são capazes de vislumbrar as implicações ulteriores do seu comportamento, compreendendo como o seu envolvimento actual nas tarefas tem significado e se relaciona com os objectivos futuros, outras pessoas preferem viver no presente, não antecipando as consequências das suas actividades presentes. Ou seja, o grau no qual as pessoas são capazes de olhar para o futuro e, assim, ver a utilidade do seu comportamento actual difere de uma pessoa para outra (Simons, Vansteenkiste, Lens, \& Lacante, 2004). Neste processo tem grande proeminência o processo de estabelecimento de projectos e objectivos que afectam as escolhas comportamentais, os esforços e a persistência nas tarefas, em especial se forem definidos pelo próprio indivíduo (Karniol \& Ross, 1996).

A importância desta dimensão temporal na vida dos indivíduos foi, por exemplo, estudada por Nuttin e Lens (1985), cujos trabalhos revelaram que, tanto os eventos passados, como os do futuro, influenciam o modo como os indivíduos pensam e consequentemente como se comportam actualmente. Com efeito, os indivíduos que estão orientados em relação ao futuro colocam uma grande ênfase no adiamento da gratificação, no planeamento, na organização, no estabelecimento de objectivos, na resistência a tentações e distracções, como também no alcance de objectivos de longo prazo (Abousselam, 2005). Este comportamento, na maioria dos casos, leva a consequências positivas na sua vida, como por exemplo o sucesso académico, menos factores de risco e mesmo a possibilidade de um estatuto sócio-económico mais elevado (Zimbardo \& Boyd, 1999).

\section{A Perspectiva Temporal de Futuro enquanto Conceito Cognitivo-Motivacional}

A perspectiva temporal de futuro (PTF) refere-se justamente às crenças ou à orientação individual em relação ao futuro, tendo em conta objectivos temporalmente distantes (Bembenutty \& Karabenick, 2003, 2004; Carvalho, 2007, 2008b; Carvalho \& Gomes, 2009; Gjesme, 1983; Husman \& Lens, 1999; Klineberg, 1968; Lessing, 1968; Simons et al., 2004,).

O conceito de PTF é geralmente descrito como uma representação ou conceptualização, em termos de tempo, de um domínio de vida particular, como seja a carreira profissional ou as relações sociais (Carvalho, 2007, 2008b; Carvalho \& Gomes, 2009; Peetsma, Hascher, \& van der Veen, 2005). Isto é, a PTF pode ser vista como uma representação em relação a um certo objecto visto num certo tempo (Stouthard \& Peetsma, 1999).

A ideia de perspectiva é aqui encarada como a representação de certos eventos ou objectos num futuro próximo ou mais distante. Portanto, trata-se do grau e o modo no qual o futuro é integrado no espaço de vida presente de um indivíduo, neste caso através de um processo motivacional de estabelecimento de objectivos e projectos (Husman \& Lens, 1999).

É um conceito caracterizado, como Peestma (2000) refere, pela extensão e pela valência. Extensão em termos de quão remota é a representação no tempo, podendo a mesma divergir de uma área para outra; valência ou relevância, no sentido da indicação do valor de um certo objecto ou domínio de vida no futuro.

Se perspectivado enquanto conceito similar ao de atitude, a PTF é também interpretada como apresentando três componentes, designadamente afectivo, cognitivo e comportamental (Rosenberg \& Hovland, 1960; Stouthard \& Peetsma, 1999), embora alguns autores (e.g., Seijts, 
1998) a considerem um constructo sobretudo cognitivo, na medida em que os indivíduos pensam sobre as suas experiências de vida e depois desenvolvem um plano de acção em termos do modo como irão agir. $\mathrm{O}$ autor defende também que a PTF é um constructo flexível, sugerindo que os indivíduos têm a capacidade de moldar as suas crenças relativamente ao seu futuro (Abousselam, 2005).

Seijts (1998) propõe ainda cinco dimensões caracterizadoras: extensão, coerência (grau de organização de eventos no leque futuro), densidade (número de eventos esperados no futuro), direccionalidade (o grau em que o individuo se percepciona como movendo-se do presente para o futuro) e afectividade (extensão em que cada um se sente gratificado por eventos futuros).

Nurmi $(1989,1993)$ apresenta um modelo conceptual da PTF, propondo a existência de três componentes: motivação, planificação e avaliação prospectiva. Relativamente à motivação, aborda a questão da extensão temporal futura, sugerindo que os motivos, interesses e metas das pessoas estão orientados para o futuro (Nuttin \& Lens, 1985), configurando o designado sistema motivacional da pessoa os valores, os motivos e os interesses.

A planificação (um constructo naturalmente mais cognitivo), o segundo componente, traduz-se na prossecução de metas/objectivos, construção de planos e execução dos mesmos. Por fim, o terceiro componente é a designada avaliação prospectiva, que se baseia no modo de adaptação associado à teoria da auto-eficácia de Bandura (1977). Inclui a avaliação do grau de controlo primário que o sujeito percebe sobre a realização das suas metas, a probabilidade de realização futura e a afectividade ligada ao futuro (Díaz-Morales, 2006).

Apesar das suas particularidades, as diversas propostas remetem para uma dupla dimensão do conceito de PTF, designadamente cognitiva e motivacional, aspectos tidos como inter-dependentes.

Nos aspectos cognitivos podemos assim mencionar (Trommsdorff, 1983): (a) a estruturação de eventos em termos da sua sequência temporal e ordem causal; (b) o objecto, a extensão, a densidade e a coerência; (c) que o conteúdo pode moldar as estruturas cognitivas, sendo aqui que entram as varáveis afectivas e motivacionais.

Relativamente aos aspectos motivacionais: (a) $\mathrm{O}$ modo como é encarado o futuro pode influenciar o comportamento e desempenha um papel importante no estudo da PTF; (b) Uma abordagem motivacional concebe a PTF como parte da actividade motivada, dirigida a objectivos, alcançados num futuro distante ou próximo.

\section{O Carácter Adaptativo da PTF: Os Exemplos na Educação e na Saúde}

Em geral, os resultados dos diversos estudos sobre o impacto que a perspectiva temporal pode ter sobre o comportamento presente têm vindo a revelar que as pes- soas com uma PTF extensa percepcionam o seu comportamento presente como mais instrumental porque $o$ mesmo as ajuda a alcançar um leque mais alargado de objectivos imediatos e futuros (aspecto cognitivo), e também valorizam mais o seu envolvimento/dedicação às tarefas actuais porque o valor antecipado do objectivo futuro é mais elevado (Simons et al., 2004). Baseado neste raciocínio, Eccles e Wigfield (1995) e Feather (1992), sugeriram que os indivíduos com uma PTF mais longa estão mais motivados em relação às suas actividades presentes, esforçam-se mais e têm um melhor desempenho em testes subsequentes (Simons et al., 2004).

Lessing (1968), por exemplo, mostrou que PTF mais extensas estavam positivamente correlacionadas com atributos considerados positivos, como sejam a inteligência, o sucesso académico, o estatuto sócio-económico e o ajustamento psicossocial em geral. Não quer dizer, naturalmente, que se trate de uma condição antecedente; contudo, parece ser clara a associação entre PTF e ajustamento.

Não é, por isso, de surpreender que a PTF seja considerada um importante factor na vida dos adolescentes e jovens adultos, em especial pela qualidade motivacional que lhe é atribuída (Stouthard \& Peetsma, 1999). Isto significa que uma PTF em relação a um certo objectivo ou objecto pode levar uma pessoa a um melhor desempenho para o atingir, já que as diferenças nesta perspectiva podem afectar qualidades motivacionais, influenciando o investimento, por parte de cada um, no seu futuro (Stouthard \& Peetsma, 1999). É neste sentido que a PTF tem uma grande relevância em contexto educativo, dimensão que importa agora analisar.

\section{Comportamentos em Contexto Educativo}

Têm sido desenvolvidos ao longo do tempo diversos estudos, cujas conclusões sublinham a relevância que a PTF assume em contexto educacional. Peetsma (2000), por exemplo, verificou que PTF diferentes, particularmente as relacionadas com a carreira escolar e profissional, são bons preditores do investimento na escola por parte dos estudantes, ou seja, quando os estudantes apresentam representações e objectivos futuros relacionados com a carreira, apresentam um maior investimento nas actividades escolares. Até porque este investimento é parte do comportamento orientado para resultados positivos e, possivelmente, para uma carreira profissional futura (Peetsma, 2000). A PTF parece, de resto, estar associada com vários factores relacionados com a carreira, como objectivos de carreira, escolhas de carreira e motivação dos estudantes (Abousselam, 2005).

Simons et al. (2004) demonstraram também que uma PTF extensa, quando associada a um aumento da instrumentalidade do comportamento actual, está associada a maior motivação, maior aprendizagem, desempenho e persistência mais intensiva nas tarefas. No mesmo sen- 
tido, Nuttin e Lens (1985) verificaram que a PTF estava positivamente correlacionada com a motivação para a aprendizagem. Do seu ponto de vista, as percepções dos estudantes acerca do futuro influenciam as suas crenças e a motivação para a aprendizagem, o que, consequentemente, tem um impacto positivo no seu nível de aquisição (Abousselam, 2005). Como Peetsma (2000) afirma, existe de facto uma ligação positiva entre o investimento na escola e as perspectivas temporais de futuro em relação à carreira escolar e profissional.

Os vários estudos, de facto, corroboram a ideia de que a PTF é uma variável explicativa e que prediz o comportamento dos estudantes, como sejam "aplicarse" ou trabalhar para a escola (De Volder \& Lens, 1982; Peetsma, 2000), estando, portanto, ligada ao sucesso.

Esta associação, por outro lado, tem sido constatada em diversos contextos societais. Por exemplo, o grau de investimento na escola por parte de estudantes na Bélgica (De Volder \& Lens, 1982) e nos Países Baixos (Peetsma, 2000) foi previsto muito bem pela extensão e valor da sua PTF.

De Volder e Lens (1982) descobriram uma correlação positiva entre a motivação de alunos do equivalente ao $11^{\circ}$ ano e a extensão da sua PTF. Estudantes mais motivados atribuíam maior valor a objectivos num futuro mais distante e também ao seu trabalho escolar para atingir esses objectivos. Há, portanto, uma inter-relação entre PTF, instrumentalidade percepcionada e a motivação dos estudantes (Simons et al., 2004). Os resultados têm vindo a indicar que os estudantes com uma PTF mais longa estão geralmente mais motivados que os estudantes com uma PTF pouco extensa. Ainda num estudo conduzido na Flandres (Bélgica), citado pelos mesmos autores, verificou-se que os estudantes que percepcionavam os seus estudos como menos instrumentais para os seus objectivos num futuro mais próximo ou distante estavam menos motivados em relação aos seus cursos.

Esta é também uma variável que é prevista como uma consequência de uma experiência frustrante (Bouffard, Lens, \& Nuttin, 1983). A PTF de indivíduos no Ruanda que não foram admitidos na universidade foi comparada com a de indivíduos que tinham sido admitidos, tendo os resultados mostrado que a dos primeiros era mais restrita em extensão que a dos que não tinham sido frustrados nas suas intenções.

Num outro estudo com estudantes universitários, verificou-se que os estudantes que tinham uma PTF estavam mais dispostos a sacrificar a gratificação presente de forma a alcançar os seus objectivos de carreira (Zimbardo \& Boyd, 1999). Tinham também competências importantes de gestão do tempo e planeamento e eram mais eficientes que os estudantes que não tinham uma PTF.

Os resultados indicaram também que os indivíduos orientados para o futuro tinham a tendência para apresentarem maiores índices de stress e preocupação, na medida em que colocavam pressão sobre si para terem desempenhos e usarem o seu tempo de modo eficiente (Zimbardo \& Boyd, 1999).

Nesta linha, já em trabalhos desenvolvidos por exemplo na África do Sul, em que se investigou o papel da perspectiva temporal na escolha de carreira em alunos do ensino secundário, verificou-se uma diferença significativa entre a perspectiva temporal média dos estudantes que tinham tomado uma decisão e a dos que não tinham tomado essa decisão, tendo os primeiros uma PTF mais longa (Abousselam, 2005).

Mais recentemente, Carvalho (2007) verificou de novo a associação existente entre PTF e sucesso, através de um estudo realizado em contexto educativo português. O estudo envolveu várias dezenas de estudantes no final da escolaridade mínima obrigatória e revelou uma interrelação muito significativa entre o sucesso na escola (avaliado através das classificações escolares e da repetência) e construção de planos de futuro. Este estudo teve ainda a particularidade de recorrer também a metodologia qualitativa, através da realização de entrevistas semiestruturadas aos alunos, opção que veiculou uma quantidade mais vasta de informação. Com efeito, o trabalho veio proporcionar, não só dados gerais sobre as interrelações existentes entre a PTF e algumas variáveis (e.g., classificações escolares, repetência de ano de escolaridade, sexo e idade), mas também elementos mais particulares e "refinados" sobre o conteúdo de muitas das perspectivas que os estudantes têm do futuro e do modo como as constroem. Neste particular, demonstrou-se a presença clara de influências de diversos agentes educativos (e.g., professores, pais, serviços de psicologia e orientação, colegas, entre outros) nas construções que os alunos desenvolvem acerca do futuro e nos objectivos que estabelecem. Estes resultados apresentam, a nosso ver, grande relevância e, além disso, revelam a utilidade da utilização de técnicas de investigação qualitativa, para além das estratégias quantitativas. Ademais - e agora numa lógica de intervenção - sustentaram a necessidade de desenvolver iniciativas no sentido da promoção de PTF nos estudantes (e.g., formação a professores sobre orientação vocacional, estabelecimento de actividades extra-curriculares, promotoras do auto-conhecimento e, consequentemente, do favorecimento de objectivos pessoais, entre outros), o que, no fundo, acaba por corresponder a práticas no âmbito de um quadro de novos desafios aos sistemas educativos (Carvalho, 2008a; Carvalho, Silva, \& Pocinho, in press).

Em todo o caso, este tipo de metodologia reiterou os resultados que têm vindo a ser encontrados, mostrando que o investimento na escola, mesmo quando os alunos não gostam à partida de algumas actividades, é levado a cabo com maior intensidade quando existem planos para o futuro. Verificou-se ainda a existência de influências de agentes educativos externos (pais, professores, colegas, media, serviços de psicologia e orientação das escolas, entre outros) na construção de tais 
planos e, especificamente, na concretização de decisões de carreira.

Motivação, Auto-regulação e Adiamento da Gratificação. Em contexto educativo, é de facto no domínio da motivação que as perspectivas temporais de futuro dos estudantes assumem uma grande expressão. Por exemplo, McInnerney, Hinkley, Dowson e van Etten (1998) verificaram que os estudantes que estavam motivados a alcançar bons resultados escolares articulavam claramente as razões pelas quais estavam na escola e descreviam a trajectória que iriam tomar depois de a deixarem. Ou seja, como Peetsma (2000) menciona, a apreciação expressa por uma pessoa em relação a um certo objecto no futuro, desempenha um papel essencial na definição do conceito de PTF como uma variável motivacional.

Nuttin (1964) classifica mesmo a PTF como o "nosso espaço motivacional primário", sendo que, desde logo, uma das primeiras publicações em torno do conceito de perspectiva de futuro - Frank (1939) - discutia as perspectivas de futuro como factores motivantes do comportamento humano.

A literatura, de facto, enfatiza o carácter motivacional da PTF (Lens, 1986; Lens \& Decruyenaere, 1991; Nuttin, 1980) e trabalhos relativamente recentes envolvendo a tradução da PTF na motivação dos estudantes (Husman \& Lens, 1999) sugerem que factores adicionais como a instrumentalidade percepcionada, motivação extrínseca versus intrínseca, valência do objectivo e auto-regulação, determinam quando tem lugar o adiamento da gratificação e como afecta a motivação dos estudantes (Bembenutty \& Karabenick, 2004).

Bembenutty e Karabenick (2004) efectuam uma descrição de cada um desses factores: Valor dos objectivos de futuro: $\mathrm{O}$ valor da recompensa adiada diminui com o aumento dos intervalos de adiamento (Mischel, 1981). Assim, as diferenças no modo como as pessoas experienciam o tempo, incluindo a sua PTF, afecta o valor de recompensas distantes. Pessoas com PTF mais longa percepcionam um dado intervalo como menos extenso do que aquelas com uma PTF curta. Porque o valor percepcionado da recompensa adiada é maior para aqueles com maior PTF, estes estão mais dispostos a adiar a gratificação (Husman \& Lens, 1999).

Instrumentalidade percepcionada: o aspecto cognitivo da PTF, refere-se à disposição para antecipar consequências de longo prazo de acções presentes. O valor de incentivo (atractividade) do alcance futuro de um objectivo (por exemplo, um grau académico) é uma função directa da PTF do estudante. Suportam esta interpretação estudos que demonstram que os estudantes que colocam um grande valor nos resultados académicos têm maior probabilidade de adiar a gratificação.

Motivação intrínseca e extrínseca: verifica-se, por exemplo, que estudantes universitários com maiores tendências para adiar a gratificação eram mais motivados intrínseca e extrinsecamente (Bembenutty \& Karabenick, 2004).
Consequências aversivas e atractivas: uma diferença importante entre o paradigma original de Mischel e contextos académicos é que os objectivos académicos futuros podem ter qualidades aversivas como também atractivas.

Auto-regulação: um aspecto importante de uma autoregulação óptima é manter-se focado na tarefa, o que envolve o uso de estratégias de aprendizagem como auto-monitorização, procurar ajuda, gestão do tempo e auto-avaliação. Os processos auto-regulatórios e crenças auto-motivacionais estão associadas com desempenhos e resultados académicos mais elevados (Zimmerman, 1998, 2000).

De facto, é razoável assumir que um sentido de propósito em relação ao futuro é importante na motivação dos indivíduos para se envolverem em actividades percepcionadas como sendo instrumentais na aquisição de resultados futuros valorizados (McInerney, 2004).

As diferenças individuais na extensão da PTF têm, portanto, consequências motivacionais (De Volder \& Lens, 1982; Lens, 1986; Raynor \& Entin, 1982). Estas diferenças afectam a força da prossecução dos objectivos actuais, por exemplo, na motivação para a aprendizagem na escola. Zaleski $(1987,1994)$ verificou que, em comparação com indivíduos com uma PTF curta, pessoas com uma PTF longa são mais persistentes a trabalhar para um objectivo e têm maior satisfação com as actividades presentes, orientadas para objectivos. Não estão só motivados por objectivos distantes mas também por sub-objectivos próximos que levam a um objectivo final num futuro mais distante.

A auto-regulação em geral e o adiamento da gratificação em particular, implicam a existência de uma PTF e demonstram a sua influência no comportamento actual (Bembenutty \& Karabenick, 2004). Ser um estudante bem sucedido depende em larga medida da resistência a tentações que trazem gratificações imediatas, de forma a aumentar a probabilidade de alcançar algo temporariamente mais remoto mas em princípio mais importante (Bembenutty \& Karabenick, 2004).

Entender o adiamento da gratificação, portanto, envolve considerar as crenças ou orientações em relação ao futuro, incluindo a perspectiva temporal de futuro dos estudantes (Husman \& Lens, 1999; Lessing, 1968).

Sendo uma função directa da PTF do indivíduo, o adiamento da gratificação é construído como uma estratégia de aprendizagem auto-regulada que, conjuntamente com crenças facilitadoras acerca do futuro, aumenta a probabilidade de completar tarefas académicas (Bembenutty \& Karabenick, 2004).

Mas, apesar da sua relação conceptual, poucos estudos examinaram directamente a relação entre o adiamento da gratificação e a PTF, sendo que outros apenas providenciaram resultados empíricos a partir dos quais essa relação pode ser inferida. Apesar de não medidos directamente, em todo caso, os dados suportam a ideia de que uma PTF extensa (por exemplo, objectivos de carrei- 
ra) está relacionada com o adiamento da gratificação por parte dos estudantes (Bembenutty \& Karabenick, 2004).

Conclui-se, assim, que uma PTF de curto prazo está associada a tendências para preferir recompensas pequenas mas imediatamente disponíveis e que ter um PTF de longo prazo está associado a preferências por recompensas maiores mas mais difundidas no tempo. Klineberg (1968) sugere mesmo que a capacidade para adiar a gratificação depende nas tendências individuais de perspectiva temporal.

\section{Promoção da Saúde e Prevenção de Comportamentos de Risco}

Apesar da PTF, quando comparada com outros conceitos, não ser muito abordada em múltiplas investigações, existem alguns estudos que analisam as interrelações entre PTF e outros domínios. Por exemplo, verifica-se a existência de alguma literatura sobre o conceito de PTF, que menciona consequências de perturbações mentais (por exemplo, depressão), como também dependência de drogas ou alcoolismo (Lilienfeld, Hess, \& Rowland, 1996; Miller, 1991).

Num estudo que investigou a relação entre a PTF e o consumo de cannabis, verificou-se que a PTF funcionou como um preditor significativo, confirmando-se o papel "protector" da PTF em consumos aditivos (Apostolidis, Fieulaine, \& Soulé, 2006). Num outro trabalho, envolvendo a relação entre a PTF e comportamentos relacionados com a saúde e segurança, que incluíam o uso de drogas, tabaco, álcool e cinto de segurança, os comportamentos sexuais e o exercício físico, verificou-se que uma PTF estava relacionada com um aumento de comportamentos protectores e com uma diminuição de comportamentos de risco (Henson, Carey, Carey, \& Maisto, 2006). Um outro exemplo é ainda a correlação significativa encontrada, numa população de jovens adultos, entre pontuações elevadas na PTF e resultados elevados para comportamentos positivos relacionados com a saúde (Mahon, Yarcheski, \& Yarcheski, 1997).

Esta associação parece, portanto, ser frequente, pelo que, como mencionam Keough, Zimbardo e Boyd (1999), a perspectiva temporal é um importante constructo, relacionado com diferenças individuais, que deve ser considerado quando se analisam comportamentos relacionados com a saúde, como o abuso de substâncias, bem como na preparação de programas de intervenção.

Ainda no domínio da saúde, podem, do mesmo modo, encontrar-se alguns exemplos da premência da PTF. Um deles é a investigação de Rothspan e Read (1999) sobre comportamentos de risco relativamente ao Vírus da Imunodeficiência Humana (VIH), em que se verificou que os jovens com uma maior orientação de futuro apresentavam uma menor probabilidade de serem sexualmente muito experientes e tinham um menor nú- mero de parceiros sexuais, apresentando, por isso, uma maior estabilidade relacional. Em contraste, os indivíduos com uma orientação de presente relacionaram-se positivamente com aquelas medidas (mais experiência sexual e maior número de parceiros sexuais). Além disso, os que apresentavam resultados mais elevados na orientação de futuro tinham maior probabilidade de usarem métodos alternativos de redução à exposição ao VIH (e.g., questionar a história sexual dos parceiros, adiar ou abster-se do sexo) (Rothspan \& Read, 1999).

\section{Conclusão}

Com o presente texto pretendeu-se fornecer um contributo para a discussão do papel do futuro no comportamento actual dos indivíduos, abordando-se, para isso, o conceito de perspectiva temporal de futuro, o qual se tentou clarificar, e debatendo-se o seu valor explicativo e preditivo em relação ao comportamento. Nesta análise, elencaram-se alguns dados provenientes de dois importantes contextos de estudo e de vivências, designadamente a educação e a saúde, este ao nível da prevenção de comportamentos de risco.

Como se pôde observar, a presença de perspectivas de futuro nos indivíduos corresponde a um elemento explicativo e mesmo preditivo do seu comportamento. Nos dois contextos mencionados têm-se, de facto, verificado resultados consistentes, que indicam que comportamentos mais adaptativos estão associados à presença, entre outros, dessas perspectivas de futuro. Isto remete, a nosso ver, para a importância da promoção destas competências junto dos indivíduos como forma de criar condições mais favoráveis à sua adaptação. Com efeito, tendo em conta os resultados já expostos, o desenvolvimento de competências de perspectivação e análise do futuro, de uma forma extensa e sólida, contribuirá para que os indivíduos - em especial estudantes e/ou jovens - possam valorizar e atribuir um maior sentido à experiência, percepcionar o valor instrumental de muitas actividades e a relação que os comportamentos presentes têm com o futuro, aspectos que ir-se-ão consubstanciar num maior investimento e na manifestação de comportamentos mais adaptativos. Isto não significa, naturalmente, que os jovens necessitem construir e estabelecer planos rígidos e objectivos extremamente operacionalizados e imutáveis para o futuro - até porque num mundo incerto e com uma imprevisibilidade crescente, tal é cada vez mais difícil. Significa outrossim, que, num mundo com estas características, estes mesmos jovens deverão desenvolver competências de perspectiva, de análise e integração de informação, que os permita situarem-se e compreenderem um eixo temporal em que se inserem, orientando-se para comportamentos mais adaptativos. Além desse valor preventivo, a presença destas capacidades promoverá uma postura activa na construção de um percurso e, portanto, a iniciativa e o 
envolvimento em actividades positivas para o desenvolvimento pessoal e profissional.

\section{Referências}

Abousselam, N. M. (2005). The moderator effect of future time perspective in the relationship between self-efficacy and risky sexual behaviour. Unpublished master's thesis, Department of Psychology, Faculty of Humanities, University of The Free State, Bloemfontein, South Africa.

Apostolidis, T., Fieulaine, N., \& Soulé, F. (2006). Future time perspective as predictor of cannabis use: Exploring the role of substance perception among French adolescents. Addictive Behaviors, 31(12), 2339-2343.

Araújo, E. R. (2005). O conceito de futuro. Actas do Seminário 'O futuro não pode começar'. Braga, Portugal: Universidade do Minho.

Bandura, A. (1977). Self-efficacy: Toward a unifying theory of behavioural change. Psychological Review, 84, 191-215.

Bembenutty, H., \& Karabenick, S. (2003). Academic delay of gratification, future goals and self regulated learning. Paper presented at the annual meeting of the American Educational Research Association, Chicago, USA.

Bembenutty, H., \& Karabenick, S. (2004). Inherent association between academic delay of gratification, future time perspective and self regulated learning: Effects of time perspective on student motivation. Educational Psychology Review, 16(1), 35-57.

Bouffard, L., Lens, W., \& Nuttin, J. R. (1983). Extension de la perspective temporelle future en relacion avec la frustration. International Journal of Psychology, 18, 429-442.

Carvalho, R. G. (2007). Perspectiva temporal de futuro em contexto educativo: Um estudo com estudantes do $9^{\circ}$ ano de uma escola rural da Madeira. Dissertação de Mestrado nãopublicada em Educação - Supervisão Pedagógica, Universidade Aberta, Lisboa, Portugal.

Carvalho, R. G. (2008a). A dinâmica relacional da intervenção dos serviços de psicologia nas escolas. Psicologia: Reflexão e Crítica, 21(1), 119-124.

Carvalho, R. G. (2008b). Future time perspective and education: Pedagogical and organizational challenges. Paper presented at the XXIX Congresso Internacional de Psicologia, Berlim, Germany.

Carvalho, R. G., \& Gomes, F. J. (2009). Perspectiva temporal de futuro e políticas de orientação. Actas do IX Congresso da Sociedade Portuguesa de Ciências da Educação: 'Educação para o Sucesso: Políticas e Actores'. Porto, Portugal: LivPsic.

Carvalho, R. G., Silva, C. S., \& Pocinho, M. (in press). Ligação entre vivências profissionais e actividades académicas: A experiência de um programa de shadowing em alunos do ensino secundário. Actas do XVII Colóquio da Association Francophone Internationale pour la Recherche Scientifique en Educacion: 'A escola e o mundo do trabalho'. Lisboa, Portugal: Association Francophone Internationale pour la Recherche Scientifique en Educacion.

De Volder, M. L., \& Lens, W. (1982). Academic achievement and future-time perspective as a cognitive-motivational concept. Journal of Personality and Social Psychology, 42, 566-571.

Díaz-Morales, J. F. (2006). Perspectiva temporal futura y ciclo vital. Anales de Psicología, 22(1), 52-59.
Eccles, J. S., \& Wigfield, A. (1995). In the mind of the actor: The structure of adolescents' achievement task values and expectancy-related beliefs. Personality and Social Psychology Bulletin, 21, 215-225.

Feather, N. (1992). Values, valences, expectations, and auctions. Journal of Social Issues, 48, 109-124.

Frank, L. K. (1939). Time perspectives. Journal of Social Philosophy, 4, 293-312.

Gjesme, T. (1983). On the concept of future time orientation: Considerations of some functions and measurement implications. International Journal of Psychology, 18, 443 461.

Henson, J. M., Carey, M. P., Carey, K. B. \& Maisto, S. A. (2006). Associations among health behaviors and time perspective in young adults: Model testing with bootstrapping replication. Journal of Behavioural Medicine, 29(2), 127-137.

Husman, J., \& Lens, W. (1999). The role of the future in student motivation. Educational Psychologist, 34(2), 113-125.

Karniol, R., \& Ross, M. (1996). The motivational impact of temporal focus: Thinking about de future and the past. Annual Review of Psychology, 47, 593-620.

Keough, K. A., Zimbardo, P. G., \& Boyd, J. N. (1999). Who's smoking, drinking, and using drugs? Time perspective as predictor of substance abuse. Basic and Applied Social Psychology, 21(2), 149-164.

Klineberg, S. L. (1968). Future time perspective and preference for delay of gratification. Journal of Personality and Social Psychology, 8, 253-257.

Lens, W. (1986). Future time perspective: A cognitivemotivational concept. In D. R. Brown \& J. Veroff (Eds.), Frontiers of motivational psychology (pp. 173-190). New York: Springer-Verlag.

Lens, W., \& Decruyenaere, M. (1991). Motivation and demotivation in secondary education: Student characteristics. Learning and Instruction, 1, 145-159.

Lessing, E. E. (1968). Demographic, developmental and personality correlates of length of future time perspective. Journal of Personality, 36, 83-201.

Lewin, K. (1935). A dynamic Theory of Personality: Selected papers of Kurt Lewin. New York: McGraw-Hill.

Lewin, K. (1942). Time perspective and morale. In G. Watson (Ed.), Civilian morale. Boston: Houghton Mifflin.

Lewin, K. (1951). Field theory in the social sciences: Selected theoretical papers. New York: Harper.

Lilienfeld, S. O., Hess, T., \& Rowland, C. (1996). Psychopathic personality traits and temporal perspective: A test of the short time horizon hypothesis. Journal of Psychopathology and Behavioral Assessment, 18, 285-314.

Mahon, N. E., Yarcheski, T. J., \& Yarcheski, A. (1997). Future time perspective and positiver health practices in young adults: An extension. Perceptual and Motor Skills, 84(3, Pt. 2), 1299-1304.

McInerney, D. (2004). A discussion of future-time perspective. Educational Psychology Review, 16(2), 141-151.

McInnerney, D. M., Hinkley, J., Dowson, M., \& Van Etten, S. (1998). Aboriginal, Anglo, and Immigrant Australian students' motivational beliefs about personal academic success: Are there cultural differences? Journal of Educational Psychology, 90, 621-629.

Miller, L. (1991). Predicting relapse and recovery in alcoholism and addiction: Neuropsychology, personality and cognitive style. Journal of Substance Abuse Treatment, 8, 277-291. 
Mischel, W. (1981). Metacognition and the rules of delay. In J. Flavell \& L. Ross (Eds.), Cognitive social development: Frontiers and possible futures (pp. 197-218). New York: Cambridge University Press.

Nurmi, J. E. (1989). Planning, motivation and evaluation in orientation to the future: A latent structure analysis. Scandinavian Journal of Psychology, 30, 64-71.

Nurmi, J. E. (1993). La perspective future dans le contexte du développement au cours de la vie. Revue Québécoise de Psychologie, 14(2), 77-97.

Nuttin, J. R. (1964). The future-time perspective in human motivation and learning. Acta Psychologica, 23, 60-82.

Nuttin, J. R. (1980). Théorie de la motivation humaine. Du besoin au projet d'action. Paris: Presses Universitaires de France.

Nuttin, J. R., \& Lens, W. (1985). Future time perspective and motivation: Theory and research method. Hillside, NJ: Lawrence Erlbaum.

Paredes, E. C., \& Pecora, A. R. (2004). Questionando o futuro: As representações sociais de jovens estudantes [Special issue]. Psicologia: Teoria e Prática, 49-65.

Peetsma, T. (2000). Future time perspective as a predictor of school investment. Scandinavian Journal of Educational Research, 44(2), 179-194.

Peetsma, T., Hascher, T., \& van der Veen, I. (2005). Relations between adolescents' self evaluations, time perspectives, motivation for school and their achievement in different countries and different ages. European Journal of Psychology of Education, 20(3), 209-225.

Raynor, J. O., \& Entin, E. E. (1982). The function of future orientation as a determinant of human behaviour in steppath theory of action. International Journal of Psychology, 18, 463-487.

Rosenberg, M. J., \& Hovland, C. I. (1960) Cognitive, affective and behavioral components of attitudes. In C. I. Hovland \& M. J. Rosenberg (Eds.), Attitude organization and change: An analysis of consistency among attitude components (pp. 1-14). New Haven, CT: Yale University Press.

Rothspan, S., \& Read, S. J. (1999). Present versus future time perspective and HIV risk among heterosexual college students. Health Psychology, 15(2), 131-134.

Seijts, G. H. (1998). The importance of future time perspective in theories of work motivation. The Journal of Psychology, 132(2), 154-168.

Simons, J., Vansteenkiste, M., Lens, W., \& Lacante, M. (2004). Placing motivation and future time perspective theory in a temporal perspective. Educational Psychology Review, 16(2), 121-139.

Stouthard, M., \& Peetsma, T. (1999). Future time perspective: Analysis of a facet designed questionnaire. European Journal of Psychological Assessment, 15(2), 99-105.

Trommsdorff, G. (1983). Future orientation and socialization. International Journal of Psychology, 18, 381-406.

Zaleski, Z. (1987). Behavioural effects of self-set goals for different time ranges. International Journal of Psychology, 22, 17-38.

Zaleski, Z. (1994). Psychology of future orientation. Lublin, Polónia: Lublin University Press.

Zimbardo, P. G., \& Boyd, J. N. (1999). Putting time in perspective: A valid, reliable individual-differences metric. Journal of Personality and Social Psychology, 77(6), 12711288.

Zimmerman, B. J. (1998). Academic studying and the development of personal skill: A self-regulatory perspective. Educational Psychologist, 33(2/3), 73-86.
Zimmerman, B. J. (2000). Attaining self-regulation: A social cognitive perspective. In M. Boekaerts, P. R. Pintrich, \& M. Zeidner (Eds.), Handbook of self-regulation (pp. 13-39). San Diego, CA: Academic Pres.
Recebido: $11 / 12 / 2008$ $1^{a}$ revisão: $31 / 03 / 2009$ $2^{a}$ revisão: 05/06/2009 $3^{a}$ revisão: 01/09/2009 Aceite final: 02/09/2009 